\title{
Effect of Heating on the Suppression of Tearing Modes in Tokamaks
}

\author{
I. G. J. Classen, ${ }^{1}$ E. Westerhof, ${ }^{1}$ C. W. Domier, ${ }^{2}$ A. J. H. Donné, ${ }^{1}$ R. J. E. Jaspers, ${ }^{1}$ N. C. Luhmann, Jr., ${ }^{2}$ H. K. Park, ${ }^{3}$ \\ M. J. van de Pol, ${ }^{1}$ G. W. Spakman, ${ }^{1}$ M. W. Jakubowski, ${ }^{4}$ and the TEXTOR team ${ }^{4}$ \\ ${ }^{1}$ FOM-Institute for Plasma Physics Rijnhuizen, Association EURATOM-FOM, \\ PO Box 1207, 3430 BE Nieuwegein, The Netherlands $*, \dagger$ \\ ${ }^{2}$ Department of Applied Science, University of California at Davis, Davis, California 95616, USA \\ ${ }^{3}$ Princeton Plasma Physics Laboratory, Princeton, New Jersey 08543, USA \\ ${ }^{4}$ Forschungszentrum Jülich GmbH, Institut für Plasmaphysik, Association EURATOM-FZJ, D-52425 Jülich, Germany*
}

(Received 17 August 2006; published 18 January 2007)

\begin{abstract}
The suppression of (neoclassical) tearing modes is of great importance for the success of future fusion reactors like ITER. Electron cyclotron waves can suppress islands, both by driving noninductive current in the island region and by heating the island, causing a perturbation to the Ohmic plasma current. This Letter reports on experiments on the TEXTOR tokamak, investigating the effect of heating, which is usually neglected. The unique set of tools available on TEXTOR, notably the dynamic ergodic divertor to create islands with a fully known driving term, and the electron cyclotron emission imaging diagnostic to provide detailed 2D electron temperature information, enables a detailed study of the suppression process and a comparison with theory.
\end{abstract}

DOI: 10.1103/PhysRevLett.98.035001

Tearing modes, and, in particular, neoclassical tearing modes (NTMs), have a deleterious effect on the performance and stability of tokamak plasmas. Larger tokamaks, like the proposed ITER tokamak, are more susceptible to the formation of NTMs. It is therefore important to develop techniques to control or suppress them and to gain understanding of the suppression process. Islands can be stabilized by driving a (helical) current perturbation inside the island region. Gyrotrons are an ideal tool for the localized generation of this current through the injection of radio frequency waves into the plasma. This current can either be directly driven noninductively by electron cyclotron current drive (ECCD) [1] or indirectly by heating the island by electron cyclotron resonance heating (ECRH) [2-4], causing a helical perturbation to the Ohmic current due to the temperature dependence of the plasma conductivity. ECCD is thought to be a more efficient way to suppress (neoclassical) islands. The tearing mode suppression by heating is often neglected. In this Letter, it will be shown that on TEXTOR the physical mechanism at work during heating can be clearly identified. It is demonstrated that also heating gives a sizeable suppression of the islands.

A set of tearing mode suppression experiments on the TEXTOR tokamak is described, that focuses on the suppression by heating (ECRH). In TEXTOR, suppression by ECRH dominates over ECCD [5] due to the low current drive efficiency (low $T_{e}$ ).

TEXTOR is a medium sized limiter tokamak with a circular plasma cross section $\left(R_{0}=1.75 \mathrm{~m}, a=0.46 \mathrm{~m}\right)$ and is ideally suited for island suppression studies due to the unique combination of available tools. With the dynamic ergodic divertor [6], islands can be created and controlled with (in contrast to other tokamaks) a fully known driving term. The gyrotron can be used to generate highly
PACS numbers: 52.55.Tn, 52.25.Fi, 52.55.Fa, 52.70.Gw

localized EC waves inside the island. Finally, the process of suppression can be observed in detail by the 2D electron cyclotron emission imaging (ECEI) diagnostic [7].

The dynamic ergodic divertor (DED) on TEXTOR is a perturbation field experiment consisting of 16 helical coils on the high field side, aligned with the $q=3$ field lines. Figure 1(a) shows the vacuum field used for the experiments described in this Letter, containing large $3 / 1$ and $2 / 1$ island chains. In the presence of a plasma, the magnetic topology changes due to shielding currents that are generated on the rational $q$ surfaces. For these experiments, this results in a plasma in which only a $2 / 1$ island is destabilized when the current in the DED coils exceeds a threshold [8]. In the underlying work, $1 \mathrm{kHz}$ ac DED currents are used, resulting in a poloidal rotation of $500 \mathrm{~Hz}$ for the $m / n=2 / 1$ island which is locked to the rotating perturbation field.

An $800 \mathrm{~kW}, 140 \mathrm{GHz}$ gyrotron is used to suppress the islands. The steerable launcher enables accurate position-

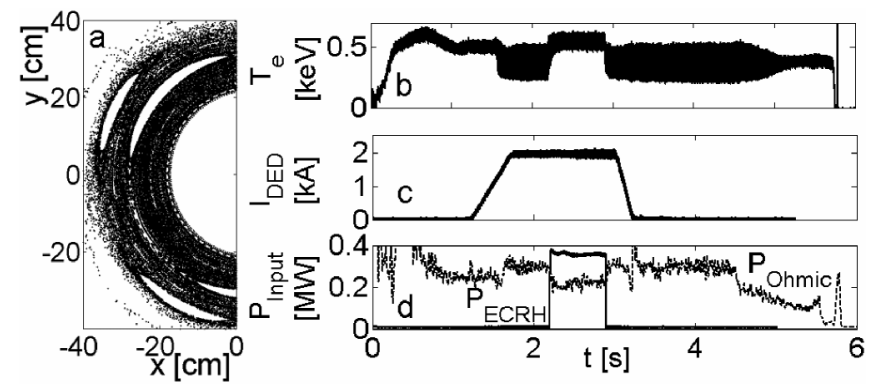

FIG. 1. Overview of a typical discharge. (a) DED vacuum magnetic field. (b) $T_{e}$ time trace from an ECEI channel just inside the island. (c) Amplitude of the DED coil currents. (d) Ohmic power and ECRH power. 
ing of the power on the resonant surface $r_{s}$ (at the high field side for these experiments). Both continuous (cw) and modulated power is possible. The radial width of the (assumed Gaussian) power deposition profile is $1.5 \mathrm{~cm}$ full width at half maximum.

The ECEI diagnostic on TEXTOR measures the electron temperature in a 2D array of 8 (radially) by 16 (vertically) observation volumes in the poloidal plane, representing about 8 by $16 \mathrm{~cm}^{2}$ in the plasma, centered on the equatorial plane. For these experiments, the field of view was adjusted to the $q=2$ surface at the low field side, and the data were sampled at $200 \mathrm{kHz}$.

Figure 1 gives an overview of a typical discharge. All discharges have a toroidal magnetic field of $2.25 \mathrm{~T}$, a toroidal plasma current of $300 \mathrm{kA}$ and a line averaged density of about $3 \times 10^{19} \mathrm{~m}^{-3}$. The DED coil current amplitude is ramped up, reaching a flat top of $2 \mathrm{kA}$ [Fig. 1(c)]. A 2/1 tearing mode is destabilized when the DED current becomes sufficiently high, as can be seen on an ECEI temperature time trace from a channel just inside the island radius as a $1 \mathrm{kHz}$ oscillation [Fig. 1(b)]. From 2.2 to $2.9 \mathrm{~s} \mathrm{ECRH} \mathrm{is} \mathrm{switched} \mathrm{on} \mathrm{[Fig.} \mathrm{1(d)],} \mathrm{either} \mathrm{cw}$ or modulated, with (for these experiments) 200, 300, or $400 \mathrm{~kW}$ of power. These power levels are comparable to the Ohmic input power. In this Letter, only data are presented for which the ECRH power was deposited directly on the resonant $q=2$ surface. The appropriate launcher position is determined from a scan of the poloidal launcher angle, searching for optimum island suppression [5].

For comprehensive visualization of the ECEI data, a poloidal reconstruction is used in which the data for one full rotation period are mapped on to a poloidal shell, assuming rigid plasma rotation. Note that the poloidal reconstruction only represents the low field side structure of the island. Figure 2 shows the reconstruction of the island during the three main stages of the suppression process. The first stage [Fig. 2(a)] is the situation in which the island has been generated and has reached a saturated

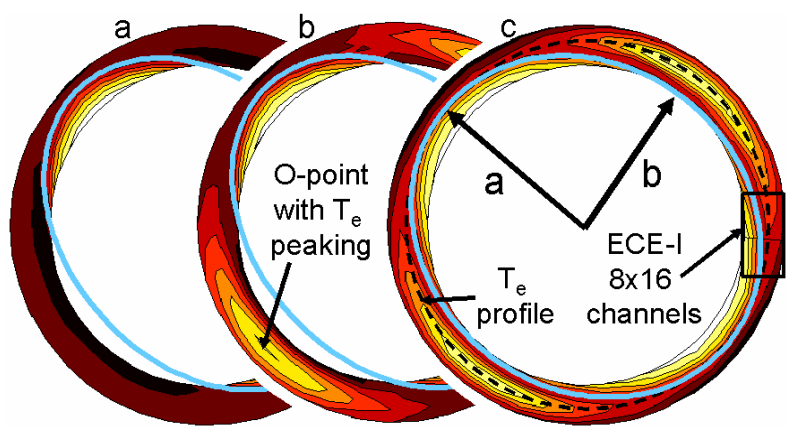

FIG. 2 (color online). Poloidal reconstructions of the island, showing the time evolution during the suppression process: (a) flat island, (b) heated island, (c) suppressed island with schematic representation of various island parameters extracted from the poloidal reconstruction. width of about $12 \mathrm{~cm}$, but before ECRH application. In this phase, the temperature profile inside the island is still flat. The hot, central plasma, approximately elliptically deformed by the island, is clearly visible. The second reconstruction [Fig. 2(b)], at about $10 \mathrm{~ms}$ after switch on of ECRH, shows a peaked $T_{e}$ profile inside the island. Although an island degrades the global confinement of the plasma, the island itself can confine energy and particles. The third stage [Fig. 2(c)] is the steady state situation long (more than $100 \mathrm{~ms}$ ) after switch on of ECRH. The island is now suppressed to about half the initial size, the central plasma is less deformed and the peaked temperature region inside the island is narrower.

An automated algorithm is used to extract the main island parameters from the reconstructed ECEI data. To determine the full island width $w$, an ellipse is fitted to the data (see Fig. 2). The difference between the major radius $a$, and minor radius $b$ of this ellipse gives the displacement of the fitted temperature contour, and is a measure for the width of the island [ $w \approx 1.5(\mathrm{a}-\mathrm{b})$ [9]]. The $O$ point position and temperature is found by looking for the temperature maximum along a line through the minor axis of the fitted ellipse. The exact position of the inner separatrix can be determined by finding the minimum temperature between the $O$ point and the ellipse. The temperature profile inside the island is evaluated on a circle through the $O$ point [dotted line in Fig. 2(c)]. In this way, the (relative) temperature profile is independent of any (relative) calibration errors, since all points on a circle are measured by the same ECEI channel(s). The temperature peaking $\Delta T_{e}$ is defined as the difference between the $O$ point and separatrix temperatures.

Figure 3 gives the typical time evolution of the full island width $w$ and the relative temperature peaking, during the suppression process. Directly after switch on of $\mathrm{ECRH}$, the temperature inside the island starts to peak, reaching a maximum peaking of typically $\Delta T_{e} / T_{e}=25 \%$ after about $10 \mathrm{~ms}$. After the $T_{e}$ peaking has formed, the island starts to shrink to typically half the initial width after about $100 \mathrm{~ms}$. The temperature peaking disappears almost immediately (within the local energy confinement time of

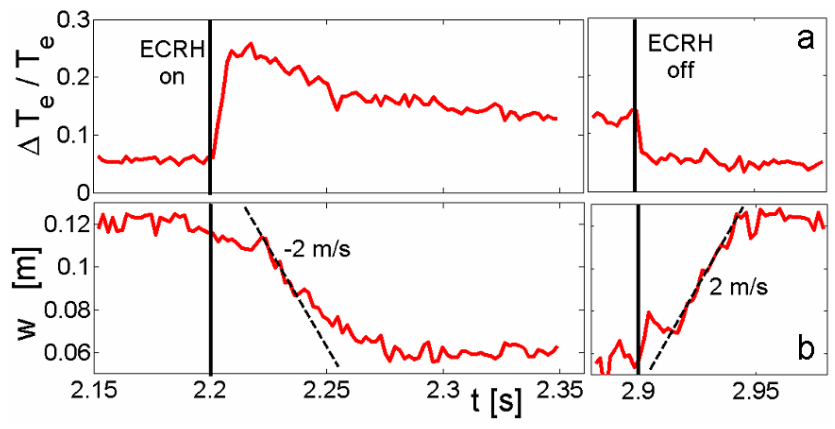

FIG. 3 (color online). Typical time evolution of (a) relative $T_{e}$ peaking and (b) island width. 
the island) after switch off of ECRH. The island then relaxes back to the initial width.

The effectiveness in which a temperature peaking forms inside an island depends on the electron heat diffusivity $\chi_{e}$ inside the island. The detailed knowledge of the temperature profile and the electron heat flux $q_{e}$ due to ECRH enables a power balance analysis. Applied to the island geometry, the standard formula for a steady state power balance $q_{e}=-n_{e} \chi_{e} \nabla T_{e}$ can be written as

$$
\left\langle q_{e}\right\rangle_{S}=-n_{e} \chi \perp, e \frac{d T_{e}}{d \Omega}\langle\nabla \Omega\rangle_{S},
$$

where \langle\rangle$_{S}$ denotes a flux surface average. The island flux surface label $\Omega=8\left(r-r_{s}\right)^{2} w^{-2}-\cos (m \xi)$ [using the helical angle $\xi=\theta-(n / m) \phi$ ] can be seen as a radial coordinate inside the island: in the $O$ point, $\Omega=-1$, on the separatrix, $\Omega=1 . T_{e}$ and $\chi_{e}$ are assumed to be flux functions. For simplicity, $n_{e}$ is taken constant over the island. Inside the island, ECRH is the only significant heating source, and is assumed to be ideally aligned on $r_{s}$ (for modulated power also centered on the $O$ point).

The numerical evaluation of Eq. (1) has been performed in the fully suppressed island stage of the discharges. Figure 4 shows the results of the power balance analysis for three discharges with different cw ECRH input power $(400,300$, and $200 \mathrm{~kW})$. The temperature profiles for these three powers are shown in Fig. 4(a). The profiles of the electron thermal diffusivity [Fig. 4(b)] show that over the largest part of the island, $\chi_{e}$ is about 1 to $1.5 \mathrm{~m}^{2} / \mathrm{s}$. Near the $O$ point, the uncertainty in the exact positioning of ECRH leads to deviations. Near the separatrix, $\chi_{e}$ rises due to the fact that close to the separatrix the electron temperature can no longer be considered a flux function [10]. A power balance analysis of the entire plasma reveals that the transport in the ambient plasma is comparable to the transport inside the island. For the ambient plasma, $\chi_{e}$ is typically $1 \mathrm{~m}^{2} / \mathrm{s}$ inside the deposition radius of ECRH and somewhat higher outside the deposition radius.

Discharges with modulated ECRH show results very similar to the $\mathrm{cw}$ results. Similar relative temperature peakings and similar transport levels are observed. The

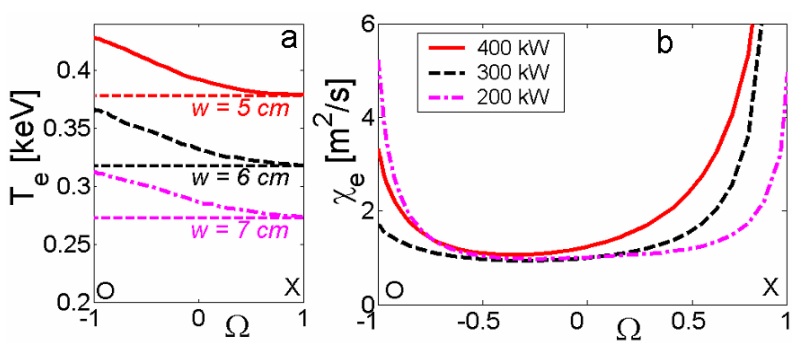

FIG. 4 (color online). Power balance inside the island for three $\mathrm{cw}$ input powers. (a) Temperature profiles inside the fully suppressed islands. Note the large effect the ECRH power has on the equilibrium (separatrix) temperature. (b) Heat diffusion coefficients inside the islands. only significant difference is that for modulated ECRH the equilibrium temperature (so the temperature of the island separatrix) rises less due to the lower total power input into the plasma. Consequently, modulation has advantages, since it does not spill power outside the island and disturbs the plasma less. Suppression works as long as there is power deposited inside the island.

The evolution of a tearing mode depends on the (helical) currents that flow inside the island region. The time evolution of the island width is governed by Eq. (2), relating the stability parameter $\Delta^{\prime}$ (the step in the poloidal flux function $\psi$ over the island region) to the total helical current inside the island region [11]

$$
\Delta^{\prime} \tilde{\psi}=2 \mu_{0} R \int_{r_{s}-w / 2}^{r_{s}+w / 2} d y \oint j_{/ /} \cos (m \xi) r_{s} d \xi
$$

If only the inductive current due to the growth of the island $d w / d t$ is taken into account, Eq. (2) results in the classical Rutherford equation [Eq. (3), skipping the last two terms] [12], where $w$ is the full island width and $\tau_{r}=$ $\mu_{0} r_{s}^{2} / \eta$ is the current diffusion time. The inclusion of other contributions to the parallel current results in modifications to the classical Rutherford equation.

For these experiments, two modifications are important. First, a destabilizing contribution $M_{\text {DED }}$ due to the shielding currents caused by the resonant external DED currents [Eq. (4), valid for strongly locked islands] [13-15]. The DED sets up a perturbed magnetic field resonant with the island, causing currents to flow inside the island region that try to compensate this external field. Second, a stabilizing contribution $M_{\mathrm{ECRH}}$ due to the perturbed Ohmic current caused by temperature perturbations inside the island [Eq. (5)] [16].

$$
\begin{aligned}
& 0.82 \tau_{r} \frac{d w}{d t}=r_{s}^{2} \Delta^{\prime}+M_{\mathrm{DED}}-M_{\mathrm{ECRH}}, \\
& M_{\mathrm{DED}}=2 m r_{s}\left(\frac{w_{\mathrm{vac}}}{w}\right)^{2}, \\
& M_{\mathrm{ECRH}}=\frac{32 q \mu_{0} r_{s}^{2}}{B_{\theta}\left|\frac{d q}{d r}\right| w^{2}} \frac{j_{\mathrm{sep}}}{T_{e, \mathrm{sep}}^{3 / 2}} \\
& \times \int_{r_{s}-w / 2}^{r_{s}+w / 2} d y \oint r_{s} d \xi T_{e}^{3 / 2} \cos (m \xi) .
\end{aligned}
$$

In principle, $\Delta^{\prime}$ is unknown for the present experiments. Before the DED is switched on, there is no $2 / 1$ island present (so the natural island is stable), but after switch off of the DED the island remains. Apparently the current profiles before and after the DED phase are not exactly the same, with $\Delta^{\prime}$ negative before the DED and positive after.

The DED term [Eq. (4)] is fully known. The vacuum island width $w_{\mathrm{vac}}=4 \mathrm{~cm}$, as can be seen from Fig. 1(a). In the DED phase of the discharges, before ECRH is switched 

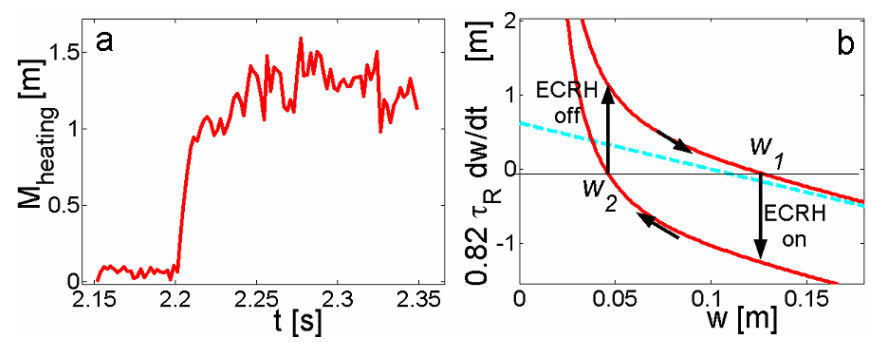

FIG. 5 (color online). The suppression process: (a) The heating term (b) Overview of the various terms in the modified Rutherford equation.

on, the saturated (plasma) island width $w_{1}$ is about $12 \mathrm{~cm}$. Saturation is reached if all terms in Eq. (3) are in balance, thereby implying $\Delta^{\prime}\left(w_{1}\right) \approx-2$.

The ECRH term $M_{\mathrm{ECRH}}$ can be evaluated numerically. The $q$ profile and current density on the separatrix $j_{\text {sep }}$ are estimated from the temperature profile. Figure 5(a) gives $M_{\mathrm{ECRH}}$ as a function of time, showing it is approximately constant at $1.2 \mathrm{~m}$.

Directly after switch on (or switch off) of ECRH, coming from a steady state situation, the heating term $M_{\mathrm{ECRH}}$ is the only one contributing to $d w / d t$. Equation (3) then predicts an initial suppression (or growth) rate of about $2 \mathrm{~m} / \mathrm{s}$, consistent with the experimentally observed values (see Fig. 3).

The relaxation to the new saturated island width $w_{2}$ (about $6 \mathrm{~cm}$ ) is determined by the balance of $M_{\mathrm{ECRH}}$ with all other terms. Figure 5(b) gives an overview of the suppression process. Plotted is the right-hand side of the modified Rutherford equation (equals $0.82 \tau_{r} d w / d t$ ) against $w$. The upper curve is composed of the $\Delta^{\prime}$ and DED terms. The lower curve includes the heating term (and is hence $1.2 \mathrm{~m}$ lower). To reproduce the observed saturated island widths, a $\Delta^{\prime}$ term of the form of the dashed line had to be introduced.

It should be noted that the stabilizing heating term in the Rutherford equation is the same for neoclassical tearing modes (NTMs) which are driven unstable by the perturbed bootstrap current instead of the DED $[10,11,16]$. In the presence of an island, the pressure gradient is removed, which results in a "hole" in the bootstrap current distribution, which gives a destabilizing contribution to the Rutherford equation. In the ITER reference scenario, bootstrap current fractions of about $30 \%$ are expected [17]. Therefore, to compensate this lost bootstrap current by the extra current due to a peaked temperature inside the NTM, a temperature peaking of about $20 \%$ is needed. Equation (6) gives the general scaling for the temperature peaking, assuming narrow islands and a power deposition width smaller than the island width

$$
\begin{array}{r}
q_{e}=-n \chi_{e} \nabla T_{e} \propto \frac{P_{\mathrm{ECRH}}}{r_{s} R}, \\
\frac{\Delta T_{e}}{T_{e}} \propto \frac{w \nabla T_{e}}{T_{e}} \propto \frac{w P_{\mathrm{ECRH}}}{r_{s} R n \chi_{e} T_{e}} .
\end{array}
$$

To get a $20 \%$ temperature peaking in ITER, Eq. (6) requires $\chi_{e}<0.2 \mathrm{~m}^{2} / \mathrm{s}$ inside the ITER islands, assuming $20 \mathrm{MW}$ of ECRH power. Even if inside the ITER islands $\chi_{e} \sim 0.5 \mathrm{~m}^{2} / \mathrm{s}$, which is equal to the expected equilibrium $\chi_{e}$ at the $q=3 / 2$ surface in ITER, the heating effect is still significant, and relaxes the gyrotron power requirements based only on current drive (ECCD).

This work, supported by the European Communities under the contract of Association between EURATOM/ FOM, was carried out within the framework of the European Fusion Program together with the U.S. Department of Energy. The views and opinions expressed herein do not necessarily reflect those of the European Commission.

*Partners in the Trilateral Euregio Cluster.

${ }^{\dagger}$ Electronic address: www.rijnh.nl

[1] R. J. La Haye, Phys. Plasmas 13, 055501 (2006).

[2] K. Hoshino et al., Phys. Rev. Lett. 69, 2208 (1992).

[3] D. C. Sing et al., Phys. Fluids B 5, 3239 (1993).

[4] D. A. Kislov et al., Nucl. Fusion 37, 339 (1997).

[5] E. Westerhof et al., Nucl. Fusion 47, 85 (2007).

[6] Special Issue, edited by K. H. Finken [Fusion Eng. Des. 37, 335 (1997)].

[7] H. Park et al., Rev. Sci. Instrum. 74, 4239 (2003).

[8] H. R. Koslowski et al., Nucl. Fusion 46, L1 (2006).

[9] I. G. J. Classen et al., in Proceedings of the 14th Joint Workshop on Electron Cyclotron Emission and Electron Cyclotron Heating, 9-12 May 2006, edited by A. Lazaros (Santorini, Greece, 2006), p. 134.

[10] R. Fitzpatrick, Phys. Plasmas 2, 825 (1995).

[11] H. R. Wilson, Trans. Fusion Sci. Technol. 45, No. 2T, 123 (2004).

[12] P. H. Rutherford, Phys. Fluids 16, 1903 (1973).

[13] R. Fitzpatrick, Nucl. Fusion 33, 1049 (1993).

[14] R. Fitzpatrick, Phys. Plasmas 5, 3325 (1998).

[15] A. W. Morris, Plasma Phys. Controlled Fusion 34, 1871 (1992).

[16] C. C. Hegna and J.D. Callen, Phys. Plasmas 4, 2940 (1997).

[17] A.C.C. Sips, Plasma Phys. Controlled Fusion 47, A19 (2005). 